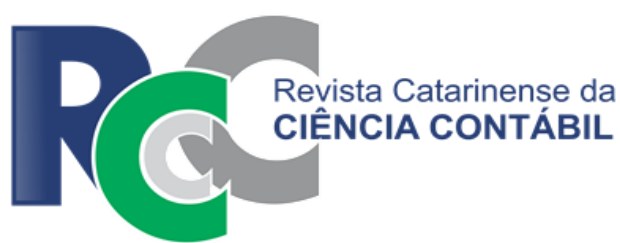

\title{
PRECIFICAÇÃO DE ATIVOS: ANÁLISE DO FATOR BOOK-TO-MARKET APÓS O DEEMED COST
}

\author{
ASSET PRICING ANALYSIS OF BOOK-TO-MARKET FACTOR AFTER \\ DEEMED COST
}

\author{
OCTÁVIO VALENTE CAMPOS \\ Universidade Federal de Minas Gerais. Endereco: Av. Antônio Carlos, \\ 6627|Pampulha| CEP 31270-010| Belo Horizonte/MG | Brasil. \\ (D) http://orcid.org/0000-0002-0354-0796 \\ octaviovc@yahoo.com.br
}

\author{
ANA CAROLINA VASCONCELOS COLARES \\ Universidade Federal de Minas Gerais. Endereço: Av. Antônio Carlos, \\ 6627|Pampulha| CEP 31270-010| Belo Horizonte/MG | Brasil. \\ (D) http://orcid.org/0000-0001-8317-752X \\ carolina_colares@hotmail.com
}

\section{RENATA TUROLA TAKAMATSU}

Universidade Federal de Minas Gerais. Endereço: Av. Antônio Carlos, 6627|Pampulha| CEP 31270-010| Belo Horizonte/MG | Brasil. (D) http://orcid.org/0000-0003-4423-9024

retakamatsu@face.ufmg.br

\author{
JOSÉ ROBERTO DE SOUZA FRANCISCO \\ Universidade Federal de Minas Gerais. Endereço: Av. Antônio Carlos, \\ 6627|Pampulha| CEP 31270-010 | Belo Horizonte/MG | Brasil. \\ (D) http://orcid.org/0000-0002-1880-5304 \\ jroberto@face.ufmg.br
}

\section{RESUMO}

A determinação do custo de capital próprio é um assunto extensivamente pesquisado e discutido em finanças, possibilitando o desenvolvimento de novos estudos correlatos. No mercado brasileiro, Noda, Martelanc e Kayo (2016) obtiveram resultados discordantes dos encontrados por Fama e French $(1995,1996)$, indicando que tais divergências podem ser explicadas pela elevada taxa de inflação. Assim, esta pesquisa busca analisar se após o deemed cost - como meio de dirimir os efeitos da inflação - os retornos obtidos pelas carteiras construídas com base no índice book-tomarket (PL/VM) são estatisticamente diferentes dos observados pelo Capital Asset Pricing Model (CAPM) e pelo indicador lucro/preço (L/P). Por meio dos resultados foi possível concluir que o índice L/P não se apresentou como mais efetivo para reconhecer ações "baratas" ou "de valor", frente ao índice book-to-market. Estes resultados são divergentes dos encontrados por Noda et al. (2016) e coincidem com os resultados encontrados por Fama e French $(1995,1996)$, evidenciando que o modelo tradicional de três fatores explica os retornos das carteiras construídas com base no 
book-to-market. Isso posto, pode-se indicar que a alta inflação histórica do mercado brasileiro, em específico para as firmas cujos ativos são mais antigos, faz com que as informações contábeis das entidades sejam menos significativas. Contudo, mensurações como as realizadas pelo deemed cost podem suavizar o impacto da inflação, concedendo maior representatividade para as informações contábeis, evidenciado assim, que práticas como o custo atribuído exercem influência nas expectativas dos usuários, conforme também verificado por Demaria e Dufour (2007) e Cerqueira, Rezende, Dalmácio e Silva (2013).

Palavras-chave: CAPM. Modelos multifatoriais. Book-to-market. Custo atribuído (deemed cost).

\section{ABSTRACT}

The determination of the cost of equity is a subject extensively researched and discussed in finance, enabling the development of new related studies. In the Brazilian market, Noda, Martelanc and Kayo (2016) obtained results discordant to those found by Fama and French (1995, 1996), indicating that such divergences can be explained by the high rate of inflation. Thus, the present research seeks to analyze whether after the deemed cost - as a means of resolving the effects of inflation - the returns obtained by the portfolios built on the book-to-market index (PL/VM) are statistically different from those observed by the CAPM by the Profit / Price indicator. By means of the results it was possible to conclude that the $L / P$ index did not present itself as more effective to recognize "cheap" or "value" stocks, compared to the book-to-market index. These results are different from those found by Noda et al. (2016) and coincide with the results found by Fama and French (1995, 1996), showing that the traditional three-factor model explains the returns of the constructed portfolios based on book-to-market. Thus, it can be pointed out that the high historical inflation of the Brazilian market, in particular for firms whose assets are older, makes the accounting information of the entities less significant. However, measurements such as those carried out by deemed cost can soften the impact of inflation, granting greater representativeness to the accounting information, thus evidenced that practices such as cost attributed influence the expectations of users, as also verified by Demaria and Dufour (2007) and Cerqueira, Rezende, Dalmácio and Silva (2013).

Keywords: CAPM. Multifactor models. Book-to-market. Assumed cost.

\section{INTRODUÇÃO}

Um campo de constantes pesquisas em finanças se dá pela busca do desenvolvimento de modelos apurados para a precificação de ativos por meio do aprimoramento Capital Asset Pricing Model (CAPM) adicionando outros fatores ao modelo, como os executados por Fama e French (1992, 1993, 2012). Dentre estes fatores, tem-se a inclusão do book-to-market (Patrimônio Líquido sobre o Valor de Mercado - PL/VM), cuja utilização teve início em trabalhos, como o que é apresentado por Stattman (1980). Neste sentido, em um mercado desenvolvido como o japonês, pesquisas demonstram resultados condizentes com a literatura ao evidenciar um beta elevado e alto indicador book-to-market (Chan, Hamao \& Lakonishok 1991; Aggarwal, Hiraki \& Rao, 1992).

Em mercados emergentes há divergência nos resultados. Trabalhos como o de Claessens, Dasgupta e Glen (1998) encontraram relação negativa entre o indicador book-to-market e os retornos, descrevendo que tal comportamento pode ser explicado pela estrutura desses mercados, na qual o regime tributário e regulamentações geram ambientes distintos, que podem influenciar o comportamento do investidor. Já trabalhos como os de Lyn e Zychomicz (2004) e Girard e Omran (2007) observaram uma relação positiva entre o indicador book-to-market e os retornos. 
No mercado brasileiro, Noda, Martelanc e Kayo (2016) aplicaram modelos de precificação de ativos financeiros em carteiras de investimentos criadas de acordo com os índices L/P e o bookto-market de empresas da B3. Apresentaram como resultados que o índice PL/VM apresenta menor eficácia para identificar ações "de valor" ou "baratas", em comparação ao índice L/P. As carteiras construídas pelo book-to-market não exibiram interceptos significativos, já as carteiras baseadas no indicador L/P apresentaram retornos anormais. Estes resultados diferem dos apresentados por Fama e French (1995, 1996). Para Noda et al. (2016), isso poderia ser explicado devido à elevada inflação brasileira, fazendo com que as informações contábeis das firmas sejam menos significativas, em específico para as firmas cujos ativos são mais antigos, já que a amostra analisada é composta pelos anos de 1995 a 2013.

A defasagem histórica na mensuração dos ativos imobilizados pode influenciar significativamente o valor contábil do patrimônio líquido, e, por consequência, os indicadores como o book-to-market. Neste sentido, de acordo com a Interpretação Técnica ICPC 10, a partir de 2010, os valores passados dos imobilizados deverão ser mensurados de acordo com os ajustes ao custo atribuído (deemed cost) pelo valor justo tratados no Pronunciamento Técnico CPC 37 e, como decorrência, no Pronunciamento Técnico CPC 43. Assim, ao coletar a amostra a partir de 2010, parte dos valores defasados devido à inflação histórica nos demonstrativos contábeis deve ser dissolvido, o que indica que para amostras a partir deste recorte, a inflação pode não exercer significante influência conforme ocorreu no trabalho de Noda et al. (2016).

Destarte, devido ainda à não existência de um método especificamente desenvolvido para mercados com altos índices inflacionários e as inconsistências encontradas no modelo de CAPM nestes mercados, busca-se por meio deste trabalho avaliar: Quais são os retornos realizados pelas carteiras brasileiras formadas com base no book-to-market e lucro/preço frente aos retornos previstos pelo CAPM após o demeed cost? Desta forma, o objetivo deste trabalho é - para uma amostra de empresas brasileiras após 2010 (incluso) - observar se os retornos realizados das carteiras baseadas no book-to-market são significativamente diferentes dos previstos pelo indicador L/P e pelo CAPM.

Isto posto, ao realizar a seleção de uma amostra brasileira e avaliar o poder explicativo do book-to-market, pode-se destacar duas vantagens: (i) verificar se medidas como a deemed cost para mensuração de ativos imobilizados exercem influência significante na análise de modelos de precificação de ativos, dado que o Brasil apresenta uma taxa média de inflação mais alta em relação aos países com mercados mais avançados e livres; e (ii) de forma geral, o mercado brasileiro, por inúmeros motivos, pode ser tido como menos líquido e eficiente, com imprecisões na precificação de ativos.

\section{REFERENCIAL TEÓRICO}

\subsection{Custo atribuído (deemed cost)}

A partir de 2010, os valores passados dos imobilizados deverão ser mensurados de acordo com os ajustes ao custo atribuído pelo valor justo, tratados no Pronunciamento Técnico CPC 37 e, como decorrência, no Pronunciamento Técnico CPC 43. Esta atribuição refere-se à uma opção aplicável somente no momento da adoção inicial, não sendo admitida a revisão da opção em períodos subsequentes (ICPC 10, 2009, item 22). As diferenças apuradas devem ser contabilizadas em contrapartida na conta de Ajustes de Avaliação Patrimonial, no Patrimônio Líquido. Este lançamento contábil é semelhante à reavaliação, já que a empresa tem a oportunidade de evidenciar o valor do ativo próximo ao seu valor econômico na data da atribuição. Portanto, a partir de 2010 os valores defasados dos imobilizados devido à inflação devem ser dissolvidos, tendo como contrapartida um aumento do Patrimônio Líquido, o que impacta no book-to-market, indicando que após esta data a inflação pode não exercer significante influência, conforme ocorreu no trabalho de Noda et al. (2016). 
No mercado francês, Demaria e Dufour (2007) pesquisaram sobre o custo atribuído para avaliar os ativos após a adoção das normas internacionais de contabilidade, constatando-se um moderado impacto sobre os preços das ações decorrente da adoção inicial ao valor justo. Ainda no contexto francês, Cormier, Demaria, Lapointe-Antunes e Teller (2009) investigaram se os incentivos gerenciais influenciam em cinco escolhas contábeis (incluindo o deemed cost) ao adotar pela primeira vez as Normas Internacionais de Relatório Financeiro (IFRS), confirmando tal hipótese.

Para o mercado brasileiro, Cerqueira et al. (2013) analisaram a relação entre o preço e o retorno das ações das empresas da Bovespa após a adoção do custo atribuído, encontrando como resultados que a adoção inicial do deemed cost impacta no preço das ações. Portanto, observa-se que a mensuração dos ativos por práticas como o custo atribuído, podem influenciar significativamente as informações contábeis, assim como as expectativas do mercado. Costa e Freitas (2014) investigaram sobre as características das companhias abertas que são determinantes da escolha pelo deemed cost para ativos imobilizados, constatando que as companhias de maior porte e mais rentáveis apresentam maior probabilidade de uso do custo atribuído. Mas, por outro lado, as empresas que são auditadas por Big 4 e que tem práticas diferenciadas de governança corporativa do "Novo Mercado" da BMF\&BOVESPA apresentam menor probabilidade de opção pelo custo atribuído.

\subsection{CAPM, Modelos multifatoriais e os retornos}

Por meio da Teoria de Diversificação de Risco desenvolvida por Markowitz (1952), Sharpe (1964) e Lintner (1965), foi desenvolvido o modelo CAPM, no qual as expectativas dos retornos dos ativos acima de uma taxa livre de risco devem ser proporcionais aos seus riscos de mercado (risco sistêmico), mensurado pelo $\beta$, multiplicado pelo prêmio esperado para uma carteira de mercado. Uma carteira de mercado pode ser formada por todos os ativos disponíveis, de forma ponderada ao seu valor. Conforme as equações 1 e $2, R j$ é o retorno desejado sobre o ativo $j ; R f$ é a taxa livre risco; $\beta$ é a medida do risco não diversificável do ativo $j$; e $R m$ é o retorno esperado do mercado para a carteira.

$$
\begin{gathered}
E\left[R_{j}\right]=R f_{t}+\beta\left(E\left[R M_{t}-R f_{t}\right)+e_{t}\right. \\
\beta=\frac{\operatorname{Cov}\left(R_{j}, R M_{t}\right)}{\operatorname{Var}\left(R M_{t}\right)}
\end{gathered}
$$

Para Levy e Roll (2010), não é possível ignorar a validade empírica deste modelo. Já para Fama e French (2004), mesmo sendo o modelo mais comumente utilizado para o cálculo do custo de capital, o CAPM nunca foi um sucesso experimental, não havendo também teste definitivo, visto que a carteira de mercado proposta pelo modelo contém ativos cujos retornos podem não ser observáveis, como: capital humano, empresas de capital fechado, ou ativos imobiliários.

Assim como apresentado por Noda et al. (2016), dado o avanço das pesquisas sobre o tema e buscando complementar o modelo do CAPM, Fama e French (1993) analisaram e desenvolveram vários modelos explicativos dos retornos de ativos nos EUA, incorporando ações (stocks) e títulos de renda fixa (bonds). Para o primeiro, os fatores de risco empregados são: (i) Rm-Rf, referindose ao fator de risco de mercado; (ii) SMB, correspondendo ao fator de risco tamanho (small minus big), ou seja, a margem de diferença entre os retornos das ações com baixa capitalização de mercado (small) e os retornos das ações com alta capitalização de mercado (big); e (iii) HML, correspondendo ao fator de risco por alto índice book-to-market, ou B/M (high minus low), o qual é apurado por meio da subtração entre os retornos das ações com alto índice B/M (high) e os retornos das ações com baixo índice B/M (small). As variáveis dependentes foram compostas de 25 carteiras com retornos acima da taxa livre de risco, Ri,t - Rft, as quais são compostas de acordo 
com a interseção de quintis de tamanho e índice book-to-market. Conforme a equação $3, a, b, s, h$ são os coeficientes da regressão e $R M t$ - Rft, SMBt e $H M L t$ são os fatores explicativos:

$$
R_{t}-R f_{t}=a+b\left(R M_{t}-R f_{t}\right)+s\left(S M B_{t}\right)+h\left(H M L_{t}\right)+e_{t}
$$

Os fatores Rm-Rf, SMB e HML explicam significativamente o retorno das carteiras e elevam consideravelmente o poder explicativo dos modelos de precificação de ativos, medido através de $\mathrm{R}^{2}$ superiores aos modelos com menos fatores, excedendo $90 \%$ na maioria das carteiras (Fama \& French, 1993). De forma complementar, Carhart (1997) propôs um modelo de quatro fatores, no qual é adicionado o fator momento de mercado, ou PRIYR, que mede a diferença de retorno entre os ativos com melhor e pior retorno no ano anterior. A equação 4 formaliza o modelo:

$$
R_{t}-R f_{t}=a+b\left(R M_{t}-R f_{t}\right)+s\left(S M B_{t}\right)+h\left(S M B_{t}\right)+p\left(P R 1 R_{t}\right)+e_{t} \quad \text { Equação (4) }
$$

Para uma amostra de 1.892 fundos mútuos de ações, no período entre 1962 e 1993 Carhart (1997) demonstrou que o fator momento possui capacidade informativa adicional aos três fatores propostos por Fama e French (1993) e também que o coeficiente $p$ é positivo, ou seja, o retorno dos ativos analisados é perdurável - ativos com retornos superiores no ano anterior demonstram uma tendência a manter retornos superiores.

Neste sentindo, para o mercado brasileiro, Noda et al. (2016) aplicaram um modelo com quatro fatores, incluindo o indicador L/P ao modelo de três fatores de Fama e French (1993). O resultado é que há um prêmio significativo para os retornos que apresentam alto índice $\mathrm{L} / \mathrm{P}$, ou value, da ordem de $1 \%$ ao mês, e também um desconto considerável de retorno para as ações com baixo índice $\mathrm{L} / \mathrm{P}$, da ordem de $0,5 \%$ ao mês. Com isso, o autor concluiu que para o mercado brasileiro, o indicador L/P é superior para explicar retornos, uma vez que não encontra prêmio ou desconto relacionado ao índice book-to-market. Tal resultado pode ser reflexo da alta inflação no Brasil, o que torna o valor contábil do patrimônio líquido das empresas defasado e, portanto, com menor significado. Também foi possível inferir que os modelos que incluem o indicador L/P, denominados HEMLE, ou high earnings minus low earnings, são os mais efetivos na eliminação dos interceptos análogos ao alfa de Jensen.

Com o objetivo de calcular os retornos, Gebhardt et al. (2001) utilizaram um modelo de lucro anormal, no qual os retornos esperados são estimados com base no valor de mercado, no lucro esperado e no valor contábil do patrimônio líquido. A equação 5 formaliza o cálculo, no qual $r$ é o custo de capital próprio, $V t$ é o valor de mercado, $B t$ é o valor contábil do patrimônio líquido e Et é o lucro líquido:

$$
V_{t}=B_{t}+\sum_{i=1}^{\infty} \frac{E_{t+i}-r B_{t+i-1}}{(1+r)^{i}}
$$

\subsection{Trabalhos empíricos em mercados emergentes}

Barry et al. (2002) verificaram a robustez do tamanho e os efeitos book-to-market em 35 mercados emergentes durante o período de 1985 e 2000. Os retornos médios das firmas com altos book-to-market excederam significativamente os retornos médios das entidades com baixos bookto-market. Por outro lado, Wang e Xu (2004), ao pesquisarem o mercado chinês entre 1990 e 2002 , encontraram que o price-to-book não foi representativo para explicar os retornos das ações.

No mercado brasileiro, Mussa, Rogers e Securato (2009) - testando o poder preditivo dos modelos CAPM em conjunto com os de três e quatro fatores - realizaram um estudo fundamentado no método proposto por Fama e MacBeth (1973), baseado em regressões que empregam os parâmetros de risco estabelecidos em um período anterior. Como conclusão, os autores 
apresentaram que os modelos testados não foram efetivos para antecipar os retornos das ações brasileiras, dada a presença significativa de alfas de Jensen.

Yoshino e Santos (2009) mediram os fatores de retorno de mercado: tamanho, PL/VM, dividend yield e P/L, por meio de regressões em painel (fully modified OLS), tendo como objetivo testar este modelo no Brasil. Para a amostra pesquisada, foi observado que estes fatores foram significativos no mercado.

Takamatsu e Fávero (2013), analisando o mercado brasileiro entre 1995 e 2010, observaram a relação entre book-to-market e os retornos futuros esperados pelas firmas, de forma que quanto maior a avaliação da empresa no mercado (exibindo um indicador book-to-market menor) maiores serão os retornos futuros da empresa. Ainda foi demonstrado que empresas maiores apresentariam menor possibilidade de insolvência, o que implicaria em menor risco e, portanto, menor prêmio retorno.

Recentemente, Noda et al. (2016) utilizaram o índice L/P como proxy para o custo do capital próprio, visando explicar os retornos das ações das empresas brasileiras, entre 1995 e 2013. Os resultados iniciais evidenciaram que as ações com baixos índices L/P têm menores retornos. As carteiras formadas pelo book-to-market não exibiram interceptos significativos, já as carteiras baseadas no indicador L/P apresentaram retornos anormais, no caso daquelas formadas por um único valor. Estes resultados diferem dos apresentados por Fama e French $(1995,1996)$. Segundo os autores, esses resultados podem decorrer devido à alta inflação histórica brasileira, gerando redução informativa no valor do patrimônio líquido, o que torna superior os modelos baseados no indicador L/P frente ao book-to-market.

\section{METODOLOGIA DA PESQUISA}

O objetivo desta pesquisa deriva dos resultados apresentados por Noda et al. (2016). Como estes autores apresentam que seus resultados diferem dos de Fama e French (1995, 1996) possivelmente devido à inflação histórica brasileira, se faz necessário observar se em um período posterior ao ajuste do custo atribuído (deemed cost) para os imobilizados - conforme a Interpretação Técnica ICPC 10 - este comportamento se dissipará e os resultados serão mais semelhantes aos apresentados por estes autores. Isso se justifica porque a amostra apresentada por Noda et al. (2016) é composta pelos anos de 1995 à 2013, período em sua maior parte por imobilizados não atualizados, o que compromete indicadores como o PL/VM, devido à subvalorizarão do PL como consequência dos valores dos imobilizados desvalorizados pela inflação histórica.

Assim, para que haja maior capacidade de comparação, a metodologia usada foi igual à apresentada por Noda et al. (2016), com exceção da amostra, como segue.

\subsection{Amostra}

A amostra utilizada foi composta por todas as empresas listadas na B3 (Bolsa, Brasil, Balcão), que possuíam dados consecutivos entre janeiro de 2010 e junho de 2017, resultando em 90 retornos mensais. As ações que não apresentam no mínimo uma das seguintes informações, foram excluídas: valor contábil do patrimônio líquido, lucro líquido, preço da ação e valor de mercado do patrimônio líquido, totalizando uma amostra final de 49 empresas.

\subsection{Variáveis e formação de carteiras}

Empregou-se o uso de regressões em séries temporais. As variáveis dependentes são os retornos mensais das carteiras de ações das firmas listadas na B3 menos à taxa livre de risco (Ri,t - Rft). Como taxa livre de risco foi usado o Certificado de Depósito Interbancário (CDI). Os fatores de risco são as variáveis explicativas do modelo, assim como feito por Fama e French (1993). 
Assim como apresentado por Noda et al. (2016), as variáveis dependentes foram formadas por meio do valor de mercado do patrimônio líquido (VM); do índice Lucro/Preço (L/P); e do book-to-market, que é o valor contábil do patrimônio líquido/valor de mercado - principal variável, cujo comportamento é analisado após o deemed cost. Como variáveis explicativas foram utilizados os fatores de risco: mercado $(R M-R F), S M B$, HML e HEMLE. As variáveis são definidas na Tabela 1, enquanto a definição das carteiras é feita na Tabela 2.

Tabela 1

\section{Metodologia de cálculo das variáveis}

\begin{tabular}{l|l}
\hline Variável & Método de cálculo \\
\hline VM & $\begin{array}{l}\text { Valor de mercado do patrimônio líquido no final de cada período, considerando o preço de cada } \\
\text { classe de ações multiplicado pelo respectivo número de ações. }\end{array}$ \\
\hline PL/VM & $\begin{array}{l}\text { Valor contábil do patrimônio líquido no final de cada período dividido pelo valor de mercado do } \\
\text { patrimônio líquido, VM, como definido anteriormente. }\end{array}$ \\
\hline L/P & O lucro por ação nos últimos 12 meses, dividido pelo preço da ação. \\
\hline RM - RF & $\begin{array}{l}\text { Retorno mensal sobre a carteira de mercado, calculada como retorno médio sobre todas as ações } \\
\text { negociadas na B3, ponderada pelo valor de mercado, menos a taxa CDI no mesmo período. }\end{array}$ \\
\hline SMB & $\begin{array}{l}\text { Small minus big: retorno mensal ponderado pelo valor de mercado da carteira S, menos retorno } \\
\text { mensal ponderado pelo valor de mercado da carteira B. }\end{array}$ \\
\hline HML & $\begin{array}{l}\text { High minus low: retorno mensal ponderado pelo valor de mercado da carteira H menos retorno } \\
\text { mensal ponderado pelo valor de mercado da carteira L. }\end{array}$ \\
\hline HEMLE & $\begin{array}{l}\text { High earnings minus low earnings: retorno mensal ponderado pelo valor de mercado da carteira } \\
\text { HE menos retorno mensal ponderado pelo valor de mercado da carteira LE. }\end{array}$ \\
\hline
\end{tabular}

Fonte: Noda, R. F., Martelanc, R., \& Kayo, E. K. (2016). O fator de risco lucro/preço em modelos de precificação de ativos financeiros. Revista Contabilidade \& Finanças, 27(70), 67-79.

Após a classificação ocorrida conforme a Tabela 1, cada ação pertence a três carteiras distintas, divididas por porte, por PL/VM e por L/P, como é apresentado na Tabela 2.

Tabela 2

\section{Classificação das ações}

\begin{tabular}{l|c|l}
\hline Critérios & Carteira & Ações \\
\hline \multirow{2}{*}{ Porte } & $\mathrm{S}$ & $50 \%$ das ações com VM menor, ou seja, ações de empresas pequenas (Small) \\
\cline { 2 - 3 } & $\mathrm{B}$ & $50 \%$ das ações com VM maior, ou seja, ações de empresas grandes (Big) \\
\cline { 2 - 3 } & $\mathrm{H}$ & $30 \%$ das ações com PL/VM maior (High) \\
\cline { 2 - 3 } & $\mathrm{M}$ & $40 \%$ das ações com PL/VM médio (Medium) \\
\cline { 2 - 3 } & $\mathrm{L}$ & $30 \%$ das ações com PL/VM menor (Low) \\
\cline { 2 - 3 } & $\mathrm{HE}$ & $30 \%$ das ações com L/P maior (High Earnings) \\
\cline { 2 - 3 } & $\mathrm{ME}$ & $40 \%$ das ações com L/P médio (Medium Earnings) \\
\cline { 2 - 3 } & $\mathrm{LE}$ & $30 \%$ das ações com L/P menor (Low Earnings)
\end{tabular}

Fonte: Noda, R. F., Martelanc, R., \& Kayo, E. K. (2016). O fator de risco lucro/preço em modelos de precificação de ativos financeiros. Revista Contabilidade \& Finanças, 27(70), 67-79.

Após este processo, as 18 carteiras finais empregadas como variáveis dependentes são fundamentadas ao mesmo tempo nos três critérios, mantendo-se composição constante pelos meses seguintes. A Tabela 3 exibe cada uma das 18, na qual cada ação irá se referir a uma única carteira.

Tabela 3

\section{Carteiras formadas}

\begin{tabular}{c|l}
\hline Carteira & Critérios \\
\hline S-L-LE & Ações pertencentes aos grupos S, L e LE \\
\hline S-L-ME & Ações pertencentes aos grupos S, L e ME \\
\hline S-L-HE & Ações pertencentes aos grupos S, L e HE \\
\hline S-M-LE & Ações pertencentes aos grupos S, M e LE \\
\hline
\end{tabular}




\begin{tabular}{c|l} 
S-M-ME & Ações pertencentes aos grupos S, M e ME \\
\hline S-M-HE & Ações pertencentes aos grupos S, M e HE \\
\hline S-H-LE & Ações pertencentes aos grupos S, H e LE \\
\hline S-H-ME & Ações pertencentes aos grupos S, H e ME \\
\hline S-H-HE & Ações pertencentes aos grupos S, H e HE \\
\hline B-L-LE & Ações pertencentes aos grupos B, L e LE \\
\hline B-L-ME & Ações pertencentes aos grupos B, L e ME \\
\hline B-L-HE & Ações pertencentes aos grupos B, L e HE \\
\hline B-M-LE & Ações pertencentes aos grupos B, M e LE \\
\hline B-M-ME & Ações pertencentes aos grupos B, M e ME \\
\hline B-M-HE & Ações pertencentes aos grupos B, M e HE \\
\hline B-H-LE & Ações pertencentes aos grupos B, He LE \\
\hline B-H-ME & Ações pertencentes aos grupos B, He ME \\
\hline B-H-HE & Ações pertencentes aos grupos B, H e HE
\end{tabular}

Fonte: Noda, R. F., Martelanc, R., \& Kayo, E. K. (2016). O fator de risco lucro/preço em modelos de precificação de ativos financeiros. Revista Contabilidade \& Finanças, 27(70), 67-79.

\subsection{Cálculo dos retornos e especificação dos modelos}

Assim como calculado por Noda et al. (2016), para cada carteira i, são calculados os retornos para cada mês $t$, Ri,t, com base nos retornos médios ponderados das ações. A equação 7 formaliza o cálculo, no qual: $V M a, t$ é o valor de mercado do $P L$ da empresa, " $a$ " no período $t ; R a, t$ é o retorno sobre a ação " $a$ " no período $t ; n$ é a quantidade de ativos que pertencem à carteira $i$; e $V M i, t$ é o valor de mercado da carteira $i$, resultante da soma do valor de mercado de todas as ações pertencentes a carteira $i$.

$$
R_{i, t}=\sum_{a=1}^{n} \frac{V M_{a, t} x R_{a, t}}{V M_{i, t}}
$$

Para apurar se as carteiras constituídas pelos índices PL/VM apresentam retornos significativamente diferentes dos previstos pelo CAPM, será utilizado o método desenvolvido por Jensen (1968) - o alfa de Jensen - tido como o comportamento anormal do ativo $i$. Conforme equação 8, Rit é o retorno sobre a carteira $i$ no mês $t$; $\alpha$ é o alfa de Jensen; e $R M t$ - RFt é o retorno de mercado que excede a taxa livre de risco no mês $t$. E, como variáveis dependentes, serão utilizados os retornos mensais sobre as carteiras de ponta formadas por um único critério $(\mathrm{H} \mathrm{e} \mathrm{L})$, assim como realizado por Noda et al. (2016).

$$
R_{i, t}-R f_{t}=\alpha_{i}+\beta_{i}\left(R M_{t}-R f_{t}\right)+e_{t}
$$

A hipótese relacionada à Equação 8 é a de que as carteiras baseadas em índices $P L / V M$ devem ter interceptos significativos - positivos para a carteira com baixo $P L / V M$ e negativos para a carteira com alto $P L / V M$. Demonstrando, tem-se:

$H 1_{0}: \alpha_{i}=0$

$H 1_{\mathrm{a} 1}: \alpha_{i}>0$, quando $i=H$

$H 1_{\mathrm{a} 2}: \alpha_{i}<0$, quando $i=L$

Serão utilizadas regressões similares às desenvolvidas por Fama e French (1993) para avaliar os modelos de precificação de ativos que contêm o fator de risco $H M L$ como variável explicativa dos retornos. Os seguintes modelos serão estimados:

Modelo 1: $R_{i, t}-R F_{t}=\alpha_{i}+b_{i}\left(R M_{t}-R F_{t}\right)+e_{i, t}$ 
Modelo 3: $R_{i, t}-R F_{t}=\alpha_{i}+b_{i}\left(R M_{t}-R F_{t}\right)+s_{i}\left(S M B_{t}\right)+h_{i}\left(H M L_{t}\right)+e_{i, t}$

Modelo 4: $R_{i, t}-R F_{t}=\alpha_{i}+b_{i}\left(R M_{t}-R F_{t}\right)+s_{i}\left(S M B_{t}\right)+k_{i}\left(H E M L E_{t}\right)+e_{i, t} \quad$ Equação (11)

Modelo 5: $R_{i, t}-R F_{t}=\alpha_{i}+b_{i}\left(R M_{t}-R F_{t}\right)+s_{i}\left(S M B_{t}\right)+h_{i}\left(H M L_{t}\right)+k_{i}\left(H E M L E_{t}\right)+e_{i, t}$ Equação (12)

Buscando maior comparação com o trabalho de Noda et al. (2016), há também a devida atenção ao fator L/P para a amostra em questão.

\section{ANÁLISE DOS RESULTADOS}

$\mathrm{Na}$ Tabela 4 é apresentado o alfa de Jensen para as carteiras baseadas em um único fator de risco, no qual o coeficiente foi positivo com $\mathrm{p}$-valor $<1 \%$ para a carteira $\mathrm{HE}$, evidenciando que as firmas com alto índice L/P apresentaram retornos significativamente acima dos previstos pelo CAPM. Estes resultados são similares aos obtidos por Costa Jr. \& Neves (2000) e Noda et al. (2016), demonstrando uma continuidade deste indicador.

O coeficiente do fator risco mercado, $\beta$, foi significativamente diferente de zero e positivo para as carteiras com critério único, estando em linha com os resultados obtidos por Fama e French (1993) e Noda et al. (2016).

Tabela 4

\section{Alfas de Jensen estimados}

\begin{tabular}{|c|c|c|c|c|c|}
\hline \multirow{2}{*}{ Carteiras } & \multicolumn{2}{|c|}{$\mathbf{a}$} & \multirow{2}{*}{$\frac{\beta}{\text { Coef. }}$} & \multirow{2}{*}{$\mathbf{R}^{2}$} & \multirow{2}{*}{ No. De obs. } \\
\hline & Coef. & $\mathbf{p}$ & & & \\
\hline$S$ & $-0,013$ & 0,002 & $0,655^{*}$ & 0,503 & 90 \\
\hline $\mathrm{B}$ & 0,003 & 0,018 & $0,837 *$ & 0,940 & 90 \\
\hline $\mathrm{H}$ & $-0,004$ & 0,416 & $1,277^{*}$ & 0,756 & 90 \\
\hline $\mathrm{L}$ & 0,007 & 0,034 & $0,916^{*}$ & 0,313 & 90 \\
\hline $\mathrm{HE}$ & 0,006 & 0,006 & $0,369 *$ & 0,880 & 90 \\
\hline 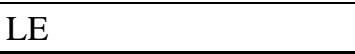 & $-0,001$ & 0,849 & $1,633^{*}$ & 0,720 & 90 \\
\hline
\end{tabular}

Fonte: Resultados da Pesquisa (2018).

A carteira (B) - grandes empresas - apresentou um coeficiente $\beta$ (beta do CAPM) próximo a 1 e um elevado $\mathrm{R}^{2}$. Como a carteira $\mathrm{B}$ é ponderada pelo valor de mercado dos ativos listados na bolsa, representando $98 \%$ de todo patrimônio do mercado brasileiro, tais resultados eram esperados. Já carteira (S) - pequenas empresas - apresenta um alfa de Jensen negativo e um menor beta. Ademais, considerando uma significância estatística de 5\%, observa-se o alfa de Jensen positivo para a carteira L, sendo este mesmo parâmetro insignificante para a carteira $\mathrm{H}$. Esses resultados divergem dos resultados de Noda et al. (2016) e se assemelham os obtidos por Fama e French $(1995,1996)$, ao indicar que ações das entidades com menor PL/VM apresentam retornos superiores comparativamente aos valores de alto PL/VM. Assim, pode-se atribuir que o deemed cost ocorrido após 2010 teve significante impacto no indicador PL/VM para as carteiras construídas por um único fator de risco.

Ratificando este resultado, observa-se também que a carteira com alto Lucro/Preço ficou acima do mercado em $0,6 \%$ a.m, já a carteira com baixo book-to-market bateu o mercado em $0,7 \%$ a.m, indicando que para as carteiras construídas por um único fator de risco, o book-to-market gera maiores rentabilidades do que o indicador $\mathrm{L} / \mathrm{P}$, sendo este um resultado contrário ao apresentado por Noda et al. (2016).

$\mathrm{Na}$ Tabela 5 é apresentado o modelo com um fator, considerando apenas o mercado, conforme a equação 9. O coeficiente $\beta$ mostrou-se positivo e significativo, como já esperado, e 
correspondente aos resultados de outros estudos brasileiros, conforme Mussa, Trovao, Santos e Famá (2007) e Noda et al. (2016). Dos 18 interceptos (alfa de Jensen - "a") das carteiras analisadas, sete se mostraram significativos, assinalando que o fator de risco mercado, apesar de relevante, não foi satisfatório na explicação dos retornos realizados pelas carteiras, opondo-se às previsões do CAPM. Devido à diferença na amostra, observa-se um acréscimo de alfas de Jensen significativos em comparação ao trabalho de Noda et al. (2016), no qual houve a presença de cinco coeficientes significativos.

Tabela 5

Parâmetros estimados para o modelo de um fator

\begin{tabular}{|c|c|c|c|c|c|c|}
\hline \multirow{2}{*}{ Carteiras } & \multicolumn{2}{|c|}{$\mathbf{a}$} & \multicolumn{2}{|l|}{$\mathbf{b}$} & \multirow{2}{*}{$\mathbf{R}^{2}$} & \multirow{2}{*}{ No. De obs. } \\
\hline & Coef. & $\mathbf{p}$ & Coef. & $\mathbf{p}$ & & \\
\hline S-L-LE & $-0,022$ & 0,002 & 1,070 & 0,000 & 0,486 & 90 \\
\hline S-L-ME & 0,010 & 0,206 & 0,332 & 0,012 & 0,069 & 90 \\
\hline S-L-HE & $-0,016$ & 0,182 & 0,625 & 0,004 & 0,092 & 90 \\
\hline S-M-LE & $-0,009$ & 0,237 & 0,329 & 0,014 & 0,067 & 90 \\
\hline S-M-ME & 0,001 & 0,854 & 0,654 & 0,000 & 0,385 & 90 \\
\hline S-M-HE & $-0,010$ & 0,046 & 0,391 & 0,000 & 0,192 & 90 \\
\hline S-H-LE & $-0,019$ & 0,027 & 0,859 & 0,000 & 0,294 & 90 \\
\hline S-H-ME & $-0,015$ & 0,056 & 0,729 & 0,000 & 0,250 & 90 \\
\hline S-H-HE & $-0,003$ & 0,381 & 0,425 & 0,000 & 0,347 & 90 \\
\hline B-L-LE & 0,011 & 0,482 & 1,965 & 0,000 & 0,377 & 90 \\
\hline B-L-ME & 0,008 & 0,045 & 0,316 & 0,000 & 0,201 & 90 \\
\hline B-L-HE & 0,012 & 0,013 & 0,490 & 0,000 & 0,309 & 90 \\
\hline B-M-LE & $-0,001$ & 0,915 & 1,536 & 0,000 & 0,576 & 90 \\
\hline B-M-ME & 0,003 & 0,291 & 0,562 & 0,000 & 0,607 & 90 \\
\hline B-M-HE & 0,007 & 0,005 & 1,012 & 0,000 & 0,866 & 90 \\
\hline B-H-LE & 1,725 & 0,839 & 0,002 & 0,000 & 0,666 & 90 \\
\hline B-H-ME & 0,006 & 0,481 & 0,756 & 0,000 & 0,255 & 90 \\
\hline B-H-HE & 0,001 & 0,927 & 0,501 & 0,000 & 0,206 & 90 \\
\hline
\end{tabular}

Fonte: Resultados da Pesquisa (2018).

Na Tabela 6 são apresentados os resultados do modelo tradicional de três fatores de Fama e French (1993), conforme equação 10. Ainda que insignificante estatisticamente em algumas carteiras, os três tiveram poder explicativo para os retornos. Uma única carteira apresentou alfa significativo com p-valor $<5 \%$, e 3 carteiras apresentaram alfa significativo com p-valor $<10 \%$. Estudos brasileiros encontraram resultados análogos a estes, como Mussa et al. (2007) e Noda et al. (2016).

Tabela 6

Parâmetros estimados para o modelo de três fatores de Fama e French

\begin{tabular}{lcccccc}
\hline \multirow{2}{*}{ Carteiras } & \multicolumn{3}{c}{ Coeficientes e p-valor entre parênteses } & & \multirow{2}{*}{$\mathbf{R}^{\mathbf{2}}$} & \multirow{2}{*}{ No. de obs. } \\
\cline { 2 - 5 } & $\mathbf{a}$ & $\mathbf{b}$ & $\mathbf{S}$ & $\mathbf{h}$ & & \\
\hline S-L-LE & $-0,006$ & 1,234 & 1,015 & 0,022 & 0,690 & 90 \\
\hline S-L-ME & $(0,355)$ & $(0)$ & $(0)$ & $(0,808)$ & & 90 \\
\hline & 0,016 & 0,347 & 0,366 & 0,057 & 0,107 & 90 \\
\hline S-L-HE & $(0,057)$ & 0,046 & $(0,06)$ & $(0,658)$ & & \\
\hline & $-0,003$ & 0,895 & 0,940 & $-0,110$ & 0,202 & 90 \\
\hline S-M-LE & $(0,828)$ & $(0,001)$ & $(0,002)$ & $(0,58)$ & & \\
\hline & 0,002 & 0,401 & 0,661 & 0,053 & 0,190 & \\
\hline S-M-ME & $(0,81)$ & $(0,017)$ & $(0,001)$ & $(0,668)$ & & \\
\hline
\end{tabular}




\begin{tabular}{|c|c|c|c|c|c|c|}
\hline & $(0,046)$ & $(0)$ & $(0)$ & $(0,052)$ & & \\
\hline \multirow[t]{2}{*}{ S-M-HE } & $-0,004$ & 0,501 & 0,388 & $-0,043$ & 0,290 & 90 \\
\hline & $(0,402)$ & $(0)$ & $(0,002)$ & $(0,598)$ & & \\
\hline \multirow[t]{2}{*}{ S-H-LE } & 0,006 & 1,117 & 1,530 & 0,021 & 0,734 & 90 \\
\hline & $(0,316)$ & $(0)$ & $(0)$ & $(0,81)$ & & \\
\hline \multirow[t]{2}{*}{ S-H-ME } & $-0,005$ & 0,781 & 0,621 & 0,066 & 0,333 & 90 \\
\hline & $(0,575)$ & $(0)$ & $(0,002)$ & $(0,605)$ & & \\
\hline \multirow[t]{2}{*}{ S-H-HE } & 0,002 & 0,478 & 0,351 & 0,011 & 0,457 & 90 \\
\hline & $(0,508)$ & $(0)$ & $(0)$ & $(0,851)$ & & \\
\hline \multirow[t]{2}{*}{ B-L-LE } & 0,026 & 2,279 & 1,019 & $-0,143$ & 0,432 & 90 \\
\hline & $(0,132)$ & $(0)$ & $(0,01)$ & $(0,583)$ & & \\
\hline \multirow[t]{2}{*}{ B-L-ME } & 0,002 & 0,711 & $-0,057$ & $-0,447$ & 0,615 & 90 \\
\hline & $(0,486)$ & $(0)$ & $(0,417)$ & $(0,000)$ & & \\
\hline \multirow[t]{2}{*}{ B-L-HE } & 0,009 & 0,639 & $-0,068$ & $-0,178$ & 0,350 & 90 \\
\hline & $(0,087)$ & $(0)$ & $(0,554)$ & $(0,022)$ & & \\
\hline \multirow[t]{2}{*}{ B-M-LE } & 0,006 & 1,263 & 0,207 & 0,341 & 0,606 & 90 \\
\hline & $(0,49)$ & $(0)$ & $(0,313)$ & $(0,014)$ & & \\
\hline \multirow[t]{2}{*}{ B-M-ME } & 0,002 & 0,676 & 0,050 & $-0,116$ & 0,641 & 90 \\
\hline & $(0,411)$ & $(0)$ & $(0,478)$ & $(0,014)$ & & \\
\hline \multirow[t]{2}{*}{ B-M-HE } & 0,004 & 1,040 & $-0,149$ & $-0,061$ & 0,876 & 90 \\
\hline & $(0,133)$ & $(0)$ & $(0,018)$ & $(0,14)$ & & \\
\hline \multirow[t]{2}{*}{ B-H-LE } & 0,009 & 0,846 & $-0,201$ & 0,927 & 0,889 & 90 \\
\hline & $(0,085)$ & $(0,000)$ & $(0,079)$ & $(0,000)$ & & \\
\hline \multirow[t]{2}{*}{ B-H-ME } & $-0,008$ & 0,815 & $-0,062$ & $-0,077$ & 0,258 & 90 \\
\hline & $(0,406)$ & $(0)$ & $(0,766)$ & $(0,579)$ & & \\
\hline \multirow[t]{2}{*}{ B-H-HE } & 0,003 & 0,570 & 0,170 & $-0,042$ & 0,220 & 90 \\
\hline & $(0,683)$ & $(0)$ & $(0,282)$ & $(0,693)$ & & \\
\hline
\end{tabular}

Fonte: Resultados da Pesquisa (2018).

A Tabela 7 contém os resultados do modelo de três fatores no qual HML é substituído pelo fator HEMLE, conforme equação 11. Neste caso aplicado ao mercado brasileiro, foi verificado comportamento semelhante ao modelo de Fama e French tradicional, no qual apresentou-se dois interceptos com p-valor $<5 \%$ e dois com p-valor $<10 \%$. No modelo tradicional de Fama e French, apresentado na Tabela 8, o coeficiente $\mathrm{h}$ foi significativo com p-valor $<10 \%$ em seis das 18 regressões, em comparação aos 7 dos 18 coeficientes k. Desta forma, observa-se que para uma amostra após o deemed cost, a diferença entre ambos os modelos se dá por um parâmetro significante, em oposição à diferença significativa apontada por Noda et al. (2016).

Tabela 7

Parâmetros estimados para o modelo de três fatores de L/P

\begin{tabular}{|c|c|c|c|c|c|c|}
\hline \multirow{2}{*}{ Carteiras } & \multicolumn{4}{|c|}{ Coeficientes e $\mathrm{p}$ valor entre parênteses } & \multirow{2}{*}{$\mathbf{R}^{2}$} & \multirow{2}{*}{ No. de obs. } \\
\hline & $\mathbf{a}$ & b & $\mathbf{S}$ & $\mathbf{k}$ & & \\
\hline \multirow[t]{2}{*}{ S-L-LE } & $-0,007$ & 1,297 & 0,994 & 0,065 & 0,692 & 90 \\
\hline & $(0.261)$ & $(0.000)$ & 0,000 & $(0.436)$ & & \\
\hline \multirow[t]{2}{*}{ S-L-ME } & 0,016 & 0,345 & 0,361 & $-0,073$ & 0,109 & 90 \\
\hline & $(0.055)$ & $(0.028)$ & $(0.058)$ & $(0.536)$ & & \\
\hline \multirow[t]{2}{*}{ S-L-HE } & $-0,002$ & 0,847 & 0,965 & 0,066 & 0,200 & 90 \\
\hline & $(0.894)$ & $(0.001)$ & $(0.001)$ & $(0.716)$ & & \\
\hline \multirow[t]{2}{*}{ S-M-LE } & 0,002 & 0,359 & 0,669 & $-0,128$ & 0,201 & 90 \\
\hline & $(0.760)$ & $(0.017)$ & $(0.000)$ & $(0.253)$ & & \\
\hline \multirow[t]{2}{*}{ S-M-ME } & 0,012 & 0,786 & 0,679 & 0,013 & 0,584 & 90 \\
\hline & $(0.014)$ & $(0.000)$ & $(0.000)$ & $(0.848)$ & & \\
\hline \multirow[t]{2}{*}{ S-M-HE } & $-0,004$ & 0,461 & 0,404 & $-0,004$ & 0,288 & 90 \\
\hline & $(0.477)$ & $(0.000)$ & $(0.001)$ & $(0.952)$ & & \\
\hline
\end{tabular}


Octávio Valente Campos, Ana Carolina Vasconcelos Colares, Renata Turola Takamatsu, José Roberto de Souza Francisco

\begin{tabular}{|c|c|c|c|c|c|c|}
\hline S-H-LE & 0,005 & 1,136 & 1,523 & 0,001 & 0,734 & 90 \\
\hline & $(0.337)$ & $(0.000)$ & $(0.000)$ & $(0.987)$ & & \\
\hline \multirow[t]{2}{*}{ S-H-ME } & $-0,007$ & 0,898 & 0,580 & 0,089 & 0,335 & 90 \\
\hline & $(0.412)$ & $(0.000)$ & $(0.003)$ & $(0.445)$ & & \\
\hline \multirow[t]{2}{*}{ S-H-HE } & 0,002 & 0,498 & 0,344 & 0,015 & 0,457 & 90 \\
\hline & $(0.557)$ & $(0.000)$ & $(0.000)$ & $(0.774)$ & & \\
\hline \multirow[t]{2}{*}{ B-L-LE } & 0,024 & 2,438 & 0,986 & 0,411 & 0,449 & 90 \\
\hline & $(0.147)$ & $(0.000)$ & $(0.010)$ & $(0.081)$ & & \\
\hline \multirow[t]{2}{*}{ B-L-ME } & 0,008 & 0,452 & 0,062 & 0,174 & 0,283 & 90 \\
\hline & $(0.062)$ & $(0.000)$ & $(0.513)$ & $(0.004)$ & & \\
\hline \multirow[t]{2}{*}{ B-L-HE } & 0,011 & 0,516 & $-0,014$ & 0,039 & 0,312 & 90 \\
\hline & $(0.029)$ & $(0.000)$ & $(0.901)$ & $(0.586)$ & & \\
\hline \multirow[t]{2}{*}{ B-M-LE } & 0,005 & 1,243 & 0,181 & $-0,454$ & 0,638 & 90 \\
\hline & $(0.534)$ & $(0.000)$ & $(0.350)$ & $(0.000)$ & & \\
\hline \multirow[t]{2}{*}{ B-M-ME } & 0,003 & 0,648 & 0,069 & 0,102 & 0,639 & 90 \\
\hline & $(0.261)$ & $(0.000)$ & $(0.315)$ & $(0.018)$ & & \\
\hline \multirow[t]{2}{*}{ B-M-HE } & 0,004 & 1,076 & $-0,153$ & 0,128 & 0,889 & 90 \\
\hline & $(0.132)$ & $(0.000)$ & $(0.009)$ & $(0.001)$ & & \\
\hline \multirow[t]{2}{*}{ B-H-LE } & 0,004 & 0,941 & $-0,318$ & $-1,012$ & 0,972 & 90 \\
\hline & $(0.130)$ & $(0.000)$ & $(0.000)$ & $(0.000)$ & & \\
\hline \multirow[t]{2}{*}{ B-H-ME } & $-0,008$ & 0,858 & $-0,067$ & 0,159 & 0,269 & 90 \\
\hline & $(0.370)$ & $(0.000)$ & $(0.740)$ & $(0.209)$ & & \\
\hline \multirow[t]{2}{*}{ B-H-HE } & 0,001 & 0,712 & 0,132 & 0,260 & 0,286 & 90 \\
\hline & $(0.900)$ & $(0.000)$ & $(0.372)$ & $(0.006)$ & & \\
\hline
\end{tabular}

Fonte: Resultados da Pesquisa (2018)

E, na Tabela 8, é apresentado os resultados do modelo com os quatro fatores, conforme equação 12. A adição do fator de risco HEMLE mostrou resultados semelhantes ao proposto pelo modelo tradicional de Fama e French. Apenas uma das 18 carteiras apresentou interceptos significativos com p-valor <0,05. Observa-se que para os dados coletados após 2010, há menor presença de alfas de Jensen significativos frente aos resultados apresentados por Noda et al. (2016) - dois interceptos significativos. Por outro lado, o trabalho destes autores apresentou maior presença de coeficientes significativos nos fatores HML e HEMLE.

Tabela 8

Parâmetros estimados para o modelo de quatro fatores

\begin{tabular}{|c|c|c|c|c|c|c|c|}
\hline \multirow{2}{*}{ Carteiras } & \multicolumn{5}{|c|}{ Coeficientes e $\mathrm{p}$ valor entre parênteses } & \multirow{2}{*}{$\mathbf{R}^{2}$} & \multirow{2}{*}{ No. de obs. } \\
\hline & $\mathbf{a}$ & b & $\mathbf{s}$ & $\mathbf{h}$ & $\mathbf{k}$ & & \\
\hline \multirow[t]{2}{*}{ S-L-LE } & $-0,005$ & 1,224 & 1,035 & 0,205 & 0,213 & 0,699 & 90 \\
\hline & $(0,429)$ & $(0)$ & $(0)$ & $(0,166)$ & $(0,117)$ & & \\
\hline \multirow[t]{2}{*}{ S-L-ME } & 0,016 & 0,351 & 0,358 & $-0,015$ & $-0,083$ & 0,109 & 90 \\
\hline & $(0,065)$ & $(0,045)$ & $(0,068)$ & $(0,944)$ & $(0,663)$ & & \\
\hline \multirow[t]{2}{*}{ S-L-HE } & $-0,003$ & 0,897 & 0,937 & $-0,139$ & $-0,034$ & 0,202 & 90 \\
\hline & $(0,821)$ & $(0,001)$ & $(0,002)$ & $(0,667)$ & $(0,909)$ & & \\
\hline \multirow[t]{2}{*}{ S-M-LE } & 0,001 & 0,412 & 0,639 & $-0,150$ & $-0,236$ & 0,206 & 90 \\
\hline & $(0,899)$ & $(0,014)$ & $(0,001)$ & $(0,449)$ & $(0,194)$ & & \\
\hline \multirow[t]{2}{*}{ S-M-ME } & 0,009 & 0,907 & 0,611 & $-0,337$ & $-0,230$ & 0,624 & 90 \\
\hline & $(0,065)$ & $(0)$ & $(0)$ & $(0,004)$ & $(0,028)$ & & \\
\hline \multirow[t]{2}{*}{ S-M-HE } & $-0,005$ & 0,505 & 0,379 & $-0,121$ & $-0,092$ & 0,295 & 90 \\
\hline & $(0,369)$ & $(0)$ & $(0,003)$ & $(0,356)$ & $(0,446)$ & & \\
\hline \multirow[t]{2}{*}{ S-H-LE } & 0,006 & 1,115 & 1,534 & 0,058 & 0,043 & 0,734 & 90 \\
\hline & $(0,307)$ & $(0)$ & $(0)$ & $(0,684)$ & $(0,741)$ & & \\
\hline \multirow[t]{2}{*}{ S-H-ME } & $-0,003$ & 0,765 & 0,655 & 0,374 & 0,358 & 0,360 & 90 \\
\hline & $(0,689)$ & $(0)$ & $(0,001)$ & $(0,069)$ & $(0,057)$ & & \\
\hline \multirow[t]{2}{*}{ S-H-HE } & 0,003 & 0,476 & 0,356 & 0,062 & 0,060 & 0,460 & 90 \\
\hline & $(0,472)$ & $(0)$ & $(0)$ & $(0,505)$ & $(0,483)$ & & \\
\hline B-L-LE & 0,029 & 2,243 & 1,096 & 0,549 & 0,805 & 0,461 & 90 \\
\hline
\end{tabular}




\begin{tabular}{|c|c|c|c|c|c|c|c|}
\hline & $(0,087)$ & $(0)$ & $(0,005)$ & $(0,184)$ & $(0,035)$ & & \\
\hline \multirow[t]{2}{*}{ B-L-ME } & 0,001 & 0,729 & $-0,094$ & $-0,776$ & $-0,383$ & 0,752 & 90 \\
\hline & $(0,792)$ & $(0)$ & $(0,103)$ & $(0)$ & $(0)$ & & \\
\hline \multirow[t]{2}{*}{ B-L-HE } & 0,008 & 0,650 & $-0,090$ & $-0,376$ & $-0,231$ & 0,382 & 90 \\
\hline & $(0,12)$ & $(0)$ & $(0,426)$ & $(0,003)$ & $(0,04)$ & & \\
\hline \multirow[t]{2}{*}{ B-M-LE } & 0,004 & 1,288 & 0,155 & $-0,127$ & $-0,545$ & 0,639 & 90 \\
\hline & $(0,638)$ & $(0)$ & $(0,434)$ & $(0,551)$ & $(0,006)$ & & \\
\hline \multirow[t]{2}{*}{ B-M-ME } & 0,003 & 0,674 & 0,054 & $-0,072$ & 0,050 & 0,643 & 90 \\
\hline & $(0,379)$ & $(0)$ & $(0,439)$ & $(0,337)$ & $(0,465)$ & & \\
\hline \multirow[t]{2}{*}{ B-M-HE } & 0,005 & 1,031 & $-0,128$ & 0,126 & 0,218 & 0,895 & 90 \\
\hline & $(0,052)$ & $(0)$ & $(0,028)$ & $(0,043)$ & $(0)$ & & \\
\hline \multirow[t]{2}{*}{ B-H-LE } & 0,005 & 0,887 & $-0,288$ & 0,151 & $-0,904$ & 0,974 & 90 \\
\hline & $(0,038)$ & $(0)$ & $(0)$ & $(0,013)$ & $(0)$ & & \\
\hline \multirow[t]{2}{*}{ B-H-ME } & $-0,006$ & 0,803 & $-0,036$ & 0,155 & 0,270 & 0,273 & 90 \\
\hline & $(0,474)$ & $(0)$ & $(0,863)$ & $(0,487)$ & $(0,187)$ & & \\
\hline \multirow[t]{2}{*}{ B-H-HE } & 0,005 & 0,543 & 0,228 & 0,475 & 0,601 & 0,357 & 90 \\
\hline & $(0,415)$ & $(0)$ & $(0,118)$ & $(0,003)$ & $(0)$ & & \\
\hline
\end{tabular}

Fonte: Resultados da Pesquisa (2018)

De forma diferente ao apresentado por Noda et al. (2016), os modelos de três e quatro fatores que utilizam o fator de risco HEMLE não fizeram diferença na eliminação dos interceptos, frente ao modelo de Fama e French tradicional. Este modelo resultou em um intercepto significativo com p-valor $<5 \%$, assim como no modelo de quatro fatores. Já o modelo de três fatores com a presença do fator de risco, HEMLE apresentou dois interceptos significativos com p-valor $<5 \%$. Tais resultados demonstram que para uma amostra coletada após os efeitos do deemed cost, os modelos de três e quatro fatores que utilizam o fator de risco HEMLE não obtiveram mais eficiência na explicação dos retornos do mercado brasileiro em comparação ao modelo clássico de três fatores.

\section{CONSIDERAÇÕES FINAIS}

Para o mercado brasileiro, o trabalho de Noda et al. (2016) indicou que o índice PL/VM apresenta baixa eficácia para identificar ações "de valor" ou "baratas". Estes resultados diferem dos apresentados por Fama e French $(1995,1996)$, de forma que aqueles autores apontaram que esta diferença poderia ser explicada devido à elevada inflação brasileira. Por outro lado, a partir de 2010, os valores passados dos imobilizados foram mensurados de acordo com os ajustes ao custo atribuído (deemed cost) pelo valor justo, indicando que para amostras após esta data a inflação histórica dos demonstrativos contábeis podem ser atenuadas. Por isso, o objetivo deste trabalho foi - após o deemed cost - observar se os retornos obtidos pelas carteiras construídas com base no book-to-market diferem significativamente dos retornos previstos pelo CAPM e pelo indicador $\mathrm{L} / \mathrm{P}$.

Para a amostra estudada, pode-se concluir que o índice L/P não se apresentou como mais efetivo para reconhecer ações "de valor", frente ao índice book-to-market. Estes resultados são divergentes dos encontrados por Noda et al. (2016) e coincidem com os resultados encontrados por Fama e French $(1995,1996)$, ao evidenciarem que o modelo de três fatores, baseadas no bookto-market, tem poder explicativo sobre os retornos das carteiras de ações.

Desta forma, pode-se indicar que a alta inflação histórica do mercado brasileiro, em específico para as firmas com ativos mais antigos, faz com que as informações contábeis das entidades sejam menos significativas. Contudo, mensurações como as realizadas pelo deemed cost podem suavizar o impacto da inflação, concedendo maior representatividade para as informações contábeis. Este resultado se alinha com os apresentados por Demaria e Dufour (2007) e Cerqueira et al. (2013), evidenciando que práticas como o custo atribuído exercem influência nas expectativas dos usuários do mercado, assim como nas informações contábeis. 
O fator de risco HML apresentou significância para explicar os retornos sobre ações nos modelos propostos. Assim, pode-se concluir também que, após o deemed cost, os modelos que empregam o fator de risco HEMLE não possuem capacidade superior para eliminar os alfas de Jensen frente aos modelos que empregam o fator HML, como o modelo tradicional de Fama e French. A partir dos resultados obtidos neste estudo é também confirmada a hipótese de que as carteiras construídas com base no book-to-market possuem interceptos representativos - positivos e significantes para a carteira com baixo book-to-market e negativos, embora sem significância, para a carteira com valor alto.

Esta pesquisa pode incentivar trabalhos que avaliem o poder explicativo do fator de risco HML em vários tipos de mercados, principalmente aqueles com a inflação menos controlada ou com a economia menos desenvolvida, principalmente em países emergentes que passaram por procedimentos contábeis como o deemed cost. Outra possível opção é testar, em maior escala, se o book-to-market é mais apropriado para os países com inflação controlada e se o índice L/P constitui um fator mais explicativo para países com alta inflação.

O mercado brasileiro gera limitações a este trabalho por se tratar de um país emergente, onde há elevada instabilidade econômica e altas taxas de juros, o que resulta em um cenário no qual o retorno de mercado é inferior à taxa livre de risco por longos períodos de tempo, tendo influência direta no fator de risco de mercado e no poder de explicação dos modelos.

\section{REFERÊNCIAS}

Aggarwal, R., Hiraki, T., \& Rao, R. P. (1992). Price/book value ratios and equity returns on the Tokyo Stock Exchange: Empirical evidence of an anomalous regularity. Financial Review, 27(4), 589-605.

Barry, C. B., Goldreyer, E., Lockwood, L., \& Rodriguez, M. (2002). Robustness of size and value effects in emerging equity markets, 1985-2000. Emerging Markets Review, 3(1), 1-30.

Carhart, M. M. (1997). On persistence in mutual fund performance. The Journal of finance, 52(1), 57-82.

Cerqueira, D. A. C., Rezende, A. J., Dalmácio, F. Z., \& Silva, J. M. da (2013). O impacto do reconhecimento do custo atribuído e da divulgação de impairment de ativos tangíveis e intangíveis (IFRS) sobre os preços e os retornos das ações das companhias brasileiras. Revista de Contabilidade do Mestrado em Ciências Contábeis da UERJ, 17(3), $5-23$.

Chan, L. K., Hamao, Y., \& Lakonishok, J. (1991). Fundamentals and stock returns in Japan. The Journal of Finance, 46(5), 1739-1764.

Claessens, S., Dasgupta, S., \& Glen, J. (1998). The cross section of stock returns: Evidence from emerging markets. Emerging Markets, Quarterly, 2, 4-13.

Comitê de Pronunciamentos Contábeis. (2009). Interpretação Técnica ICPC 10: Interpretação sobre a Aplicação Inicial ao Ativo Imobilizado e à Propriedade para Investimento dos Pronunciamentos Técnicos CPCs 27, 28, 37 e 43. Brasília, dez.

Comitê de Pronunciamentos Contábeis. (2009). CPC 37: adoção inicial das Normas Internacionais de Contabilidade. Brasília, dez.

Comitê de Pronunciamentos Contábeis. (2010). CPC 43: Adoção Inicial dos Pronunciamentos Técnicos CPCs 15 a 41. Brasília, dez. 
Cormier, D., Demaria, D., Lapointe-Antunes, P., \& Teller, R. (2009). First-Time Adoption of IFRS, Managerial Incentives and Value- Relevance: Some French Evidence. Journal of International Accounting Research, American Accounting Association, 8(2), 1-22.

Costa, F. M., \& Freitas, K. C. (2014). Escolhas contábeis na adoção inicial das normas internacionais de contabilidade no Brasil: direcionadores da aplicação do custo atribuído para ativos imobilizados. Revista Contabilidade Vista \& Revista, 25(3), 38-56.

Costa Jr., N., \& Neves, M. B. (2000). Variáveis fundamentalistas e os retornos das ações. Revista Brasileira de Economia, 54(1), 123-137.

Demaria, S., \& Dufour, D. (2007). First time adoption of IFRS, Fair value option, Conservatism: Evidences from French listed companies. In 30 ème colloque de l'EAA (p. 24p).

Fama, E. F., \& French, K. R. (1992). The cross-section of expected stock returns. The Journal of Finance, 47(2), 427-465.

Fama, E. F., \& French, K. R. (1993). Common risk factors in the returns on stocks and bonds. Journal of financial economics, 33(1), 3-56.

Fama, E. F., \& French, K. R. (1995). Size and book-to-market factors in earnings and returns. The journal of finance, 50(1), 131-155.

Fama, E. F., \& French, K. R (1996). Multifactor explanations of asset pricing anomalies. Journal of Finance, 51(1), 55-84.

Fama, E. F., \& French, K. R. (2004). The capital asset pricing model: Theory and evidence. The Journal of Economic Perspectives, 18(3), 25-46.

Fama, E. F., \& French, K. R. (2012). Size, value, and momentum in international stock returns. Journal of financial economics, 105(3), 457-472.

Fama, E. F., \& Macbeth, J. D. (1973). Risk, return, and equilibrium: Empirical tests. Journal of political economy, 81(3), 607-636.

Gebhardt, W. R., Lee, C., Swaminathan, B. (2001). Toward an implied cost of capital. Journal of accounting research, 39(1), 135-176.

Girard, E., \& Omran, M. (2007). What are the risks when investing in thin emerging equity markets: Evidence from the Arab world. Journal of International Financial Markets, 17(1), 102-123.

Jensen, M. C. (1968). The performance of mutual funds in the period 1945-1964. The Journal of finance, 23(2), 389-416.

Levy, M., \& Roll, R. (2010). The market portfolio may be mean/variance efficient after all. Review of Financial Studies, 23(6), 2464-2491.

Lintner, J. (1965). Security prices, risk, and maximal gains from diversification. The journal of finance, 20(4), 587-615.

Lyn, E. O., \& Zychowicz, E. J. (2004). Predicting stock returns in the developing markets of Eastern Europe. The Journal of investing, 13(2), 63-71. 
Markowitz, Harry. (1952). Portfolio selection. The journal of finance, 7(1), 77-91.

Mussa, A., Trovao, R., Santos, J. O., \& Famá, R. (2007). A estratégia de momento de Jegadeesh e Titman e suas implicações para a hipótese de eficiência do mercado acionário brasileiro. Seminários em Administração, 10, 2007.

Mussa, A., Rogers, P., \& Securato, J. R. (2009). Modelos de retornos esperados no mercado brasileiro: testes empíricos utilizando metodologia preditiva. Revista de Ciências da Administração, 11(23), 192.

Noda, R. F., Martelanc, R., \& Kayo, E. K. (2016). O fator de risco lucro/preço em modelos de precificação de ativos financeiros. Revista Contabilidade \& Finanças, 27(70), 67-79.

Sharpe, William F. (1964). Capital asset prices: A theory of market equilibrium under conditions of risk. The journal of finance, 19(3), 425-442.

Stattman, D. (1980). Book values and stock returns. The Chicago MBA: A journal of selected papers, 4(1), 25-45.

Takamatsu, R. T., \& Fávero, L. P. L. (2013). Accruals, persistence of profits and stock returns in Brazilian public companies. Modern Economy, 4(02), 109.

Yoshino, J. A., \& Santos, E. B. (2009). Is the CAPM Dead or Alive in the Brazilian Market? Review of Applied Economics, 5(1-2).

Wang, F., \& Xu, Y. (2004). What determines Chinese stock returns? Financial Analysts Journal, 6(6), 65-77. 